\title{
MEDIAÇÃO TECNOLÓGICA E CARACTERIZAÇÃO DE MOOCS: UMA ABORDAGEM DA CÁTEDRA VIRTUAL INNOVATIC 2.0
}

\author{
MEDIACIÓN TECNOLÓGICA Y CARACTERIZACIÓN DE LOS MOOC: UNA \\ APROXIMACIÓN DESDE LA CÁTEDRA VIRTUAL INNOVATIC 2.0
}

\section{TECHNOLOGICAL MEDIATION AND CHARACTERIZATION OF MOOCS: AN APPROACH TO THE CÁTEDRA VIRTUAL INNOVATIC 2.0}

\author{
Rubén EDEL-NAVARRO ${ }^{1}$ \\ Germán RUIZ-MÉNDEZ ${ }^{2}$ \\ Genaro Aguirre AGUILAR ${ }^{3}$
}

RESUMO: São apresentados os resultados da implementação de um curso massivo on-line aberto ou MOOC, destinado ao treinamento de habilidades para inovação de uma população de 28612 estudantes de nível superior no contexto de instituições de ensino mexicanas. A pesquisa foi realizada a partir de uma abordagem metodológica mista para determinar as características descritivas e os resultados do curso on-line, com base na análise dos dados e informações dos participantes, bem como na pesquisa de satisfação aplicada para identificar os perfis sociodemográficos, as condições de usabilidade e os formatos da experiência de aprendizagem dos jovens durante o treinamento em habilidades de inovação. Os resultados permitiram a revisão histórica do MOOC, através da caracterização de seus usuários, suas principais contribuições no ensino superior, seu escopo e cobertura, bem como o grau de satisfação do usuário.

PALAVRAS-CHAVE: Mediação tecnológica. TIC. MOOC. Aprendizagem on-line. Educação a distância.

RESUMEN: Se presentan los resultados de la implementación de un curso abierto masivo en línea o MOOC (por sus siglas en inglés) dirigido a la formación de habilidades para la innovación de una población de 28612 estudiantes del nivel superior en el contexto de instituciones educativas mexicanas. La investigación se realizó desde un enfoque metodológico mixto para determinar, tanto las características descriptivas, como los resultados del curso en línea, lo anterior a partir del análisis de los datos e información de los participantes, así como por la encuesta de satisfacción aplicada en forma aleatoria para identificar los perfiles sociodemográficos, las condiciones de la usabilidad y los formatos de la experiencia de aprendizaje de los jóvenes durante su formación en habilidades para la innovación. Los resultados permitieron la reseña histórica del MOOC, a través de la

\footnotetext{
${ }^{1}$ Universidad Veracruzana, Veracruz - Veracruz - México. Professor-Pesquisador da Faculdade de Pedagogia. ORCID: http://orcid.org/0000-0002-7066-4369. E-mail: redel@uv.mx

${ }^{2}$ Universidad Nacional Autónoma de México (UNAM), Ciudad de México - México. Professor da UNAM, Coordenador ECESELI-UDUAL. ORCID: https://orcid.org/0000-0003-3172-8689. E-mail: german.ruiz@udual.org

3 Universidad Veracruzana, Boca del Río - Veracruz - México. Professor-Pesquisador da Faculdade de Comunicação. ORCID: https://orcid.org/0000-0001-5223-9783. E-mail: geaguirre@uv.mx
} 
caracterización de sus usuarios, de sus contribuciones principales en la educación superior, de su alcance y cobertura, así como del grado de satisfacción de los usuarios.

PALABRAS CLAVE: Mediación tecnológica. TIC. MOOC. Aprendizaje en línea. Educación a distancia.

ABSTRACT: The results of the implementation of a massive open online course or MOOC are presented, aimed at training skills for innovation in a population of 28612 higher education students in the context of Mexican educational institutions. The research was made out of a mixed methodological approach to determine the descriptive characteristics and the results of the online course, based on the analysis of the data and information of the participants, as well as on the applied satisfaction survey to identify the sociodemographic profiles, the conditions of usability and the formats of the learning experience of young people during training in innovation skills. The results allowed the historical review of the $M O O C$, through the characterization of its users, their main contributions to higher education, its scope and coverage, as well as the user satisfaction degree.

KEYWORDS: Technological mediation. ICT. MOOC. Online learning. Distance education.

\section{Introdução}

A educação on-line e a distância se tornaram componentes indispensáveis para o ensino formal nas universidades ao longo dos últimos anos. Para tais efeitos, foram desenvolveram múltiplos formatos, plataformas e ferramentas que são gerados a partir dos avanços das tecnologias da informação e comunicação (TIC), bem como do aumento nas coberturas e no acesso a internet, o que levou a um aumento exponencial no ensino universitário (AREA, 2018).

Isso tem permitido o desenvolvimento de plataformas ou ambientes virtuais de aprendizagem que permitem o trabalho colaborativo entre professores e alunos, fortalecendo o ensino tradicional com novos desenvolvimentos e inovações na educação.

Outra componente que exige a formação de jovens em instituições de ensino superior corresponde às temáticas da inovação, empreendedorismo e criatividade para desenvolver bens e serviços com base nos conhecimentos adquiridos ao longo da formação académica, para os quais grande parte das universidades tem fortalecido seus programas educacionais nesses temas, como um componente do desenvolvimento de habilidades para os jovens.

Combinando estes dois aspectos, virtualidade e conteúdo de inovação, os resultados do curso online denominado Cátedra Virtual Innovatic 2.0, que consistiu em um curso chamado MOOC, para o desenvolvimento de habilidades em inovação dos jovens com o objetivo de 
documentar as contribuições e alcances que a educação on-line apresenta no apoio à formação de jovens do nível superior.

\section{Educação e revolução tecnológica}

Nas últimas décadas, as TIC têm se configurado como a chamada revolução da aprendizagem (STIGLITZ; GREENWALD, 2014), na qual a economia da sociedade gira em torno dos desenvolvimentos tecnológicos e da economia digital.

Na educação a situação não é diferente, uma vez que o conhecimento e a inovação são componentes que têm predominado na educação on-line e a distância, juntamente com os desenvolvimentos tecnológicos baseados na internet.

Neste sentido, nos últimos 10 anos, a revolução do conhecimento tem penetrado nas salas de aula em grande parte das universidades e instituições educacionais do mundo todo, os entornos virtuais de aprendizagem (EDEL; NAVARRO, 2015) foram um fator chave pelos quais alunos e professores promovem uma aprendizagem colaborativa para potencializar o escopo e a cobertura educacionais.

Desta forma, os desenvolvimentos tecnológicos baseados na internet permitiram que o e-learning abrisse espaço para a criação de plataformas de aprendizagem como moodle, blackboard e outros projetos baseados nos chamados recursos abertos (open source). Tais plataformas configuram dois componentes essenciais para a gestão de cursos e programas de formação on-line: LMS (learning management system) e CMS (content management system).

É dessa forma que a educação e e-learning têm transitado por componentes geracionais graças à revolução do software (CASTAÑO; GARAY; THEMISTOKLEOUS, 2018), os modelos educacionais têm moldado seus componentes através do enfoque 1.0 ao 3.0, conforme a web avança para suas imersões nos âmbitos educacionais, enquanto conteúdos, atividades de aprendizagens, papeis institucionais, envolvimento do estudante e da tecnologia.

Com base na literatura analisada, são identificadas as seguintes modalidades na distribuição da aprendizagem online (BATES, 2015):

1. Ensino em sala de aula

2. Aprendizagem semipresencial

3. Aprendizagem híbrida ou flexível

4. Aprendizagem totalmente on-line, sem encontros presenciais. 
No quarto componente desenvolvido por Bates, se encontra inserido o campo técnoeducacional, que considera a criação e distribuição de conteúdos educacionais, o qual abriu espaço para o surgimento dos denominados MOOC (massive online open courses) como uma ferramenta para a abertura do conhecimento aberto das universidades.

O termo MOOC, consolidado por Dave Cormier e Bryan Alexander em 2008, permitiu a criação do primeiro curso on-line, este teve como título Connectivism and Connective Knowledge organizado por George Siemens e Stephen Downes na Universidade de Manitoba, Canadá, em agosto do presente ano.

O curso com 12 semanas de duração, contou com a participação de 2300 estudantes a nível mundial, o qual marcou um precedente importante para o ensino à distância. $\mathrm{O}$ segundo curso MOOC desenvolvido foi nomeado Introduction to Artificial Intelligence, por um professor da Universidade de Stanford e Peter Norvig, diretor de pesquisa do Google, que, ao longo do ano de 2011, teve 160 mil participantes inscritos no contexto mundial.

O seguinte curso desta magnitude foi Circuits and Electronics organizado em 2012, pelo professor Anant Agarwal do Massachusetts Institute of Technology (MIT) em sua plataforma MITx, com mais de 120 mil participantes. Posteriormente, os professores Andrew $\mathrm{Ng}$ e Daphne Koller da Universidade de Stanford, fundaram a plataforma Coursera e começaram a oferecer cursos a partir de abril de 2012.

Como afirma Zubieta (2015), a tendência dos MOOC evoluiu por responder às demandas de conhecimento, habilidades e atitudes que os indivíduos devem se atender para prosperar em uma economia competitiva como a atual, bem como por ser um modelo que permite obter novas habilidades.

Os elementos pedagógicos do modelo MOOC estão baseados na escola da conectividade, pois fundamenta que "o conhecimento pessoal é composto por uma rede alimentada por instituições de ensino, que por sua vez agem com a mesma rede, gerando uma nova aprendizagem ao indivíduo" (SIEMENS apud ZUBIETA, 2015, p. 178).

A oferta do $M O O C$ demostrou que há uma necessidade na cidadania mundial de acessar uma educação flexível, adaptável, especializada e de qualidade (VALVERDE, 2014).

Da mesma forma, sete tipos de MOOC são distinguidos (CLARK, 2013, p. 2-11):

- TransferMOOC. Cursos já disponíveis que são transferidos para o formato MOOC.

- MadeMOOC. Mais inovadores, fazem uso eficaz de vídeos e material interativo; têm um nível de qualidade superior.

- AsynchMOOC. Não há data fixa de início, nem de finalização e apresenta flexibilidade nos prazos de entrega. 
- AdaptuveMOOC. Proporciona experiências de aprendizagem personalizadas, baseadas em avaliações dinâmicas e no uso de uma grande quantidade de dados coletados no curso (learning analytics).

- GroupMOOC. O fator principal é a colaboração que existe nos pequenos grupos.

- ConnectivistMOOC. Ênfase na conexão entre uma rede de participantes.

- MiniMOOC. Muito mais limitados com relação ao conceito tradicional.

No campo da educação on-line, são reconhecidos quatro tipos de participantes em um MOOC:

a) Ouvintes ou participantes passivos: estudantes que acessam a todos os vídeos com as exposições dos docentes, mas apenas utilizam as avaliações do curso ou participam nos fóruns ou outras atividades de comunicação.

b) Comprometidos ou participantes ativos: alunos que utilizam todos os materiais didáticos, realizam todas ou quase todas as avaliações que o curso oferece e se comprometem participado dos fóruns e das redes sociais.

c) Desconectados ou inconstantes: estudantes que realizam as avaliações no início do $M O O C$, mas que depois apenas acessam algumas aulas ou desaparecem por completo do curso.

d) Provadores e exploradores: alunos que exploram superficialmente o curso mediante ao uso de alguns vídeos (Conole apud Torres; Gago, 2014, p. 15).

Existem fatores que favorecem a evolução dos $M O O C$, quais sejam;

- A maturidade técnica e pedagógica alcançada pelos sistemas de formação online (e-learning).

- O entusiasmo geral para a formação a distância apoiada pelo uso de plataformas learning management systems (LMS).

- A tendência para os movimentos e materiais abertos: penetração da Web 2.0 no ensino superior, disseminação de Open CourseWare (OCW) e a produção de Recursos Educacionais Abertos (REA) (MARTÍNEZ; RODRÍGUEZ; GARCÍA apud ZUBIETA, 2015, p. 179).

Reconhece-se que as universidades têm unido esforços, capacidades e iniciativas (VALVERDE, 2014) para dar lugar a diferentes associações acadêmicas que elaboram e produzem MOOC através de diversas plataformas tecnológicas, criando assim um fator positivo na formação de uma oferta educacional de maior qualidade, mais ampla e com melhores expectativas de chegar a um maior número de cidadãos.

Os MOOC permitiram demonstrar na educação superior, que é necessária uma transformação orientada para um desenho modular, descentralizado, baseado em projetos, 
dinâmico e adaptável, que exclua o desenho rígido, padronizado e inflexível (VALVERDE, 2014).

A educação a distância permitiu que a sociedade mexicana tivesse alternativas que permitiam acessar a programas educacionais, com tendência ao global, a democratizar o ensino superior e o aproveitamento das possibilidades que outorgam as TIC nos diversos processos educacionais (GARDUÑO, 2008).

\section{A revolução internacional dos $M O O C$ e o caso do México}

Em 2 de maio do ano de 2012, o MIT e a Universidade de Harvard, anunciaram o projeto conjunto da plataforma de MOOC Edx (https://www.edx.org), como uma organização sem fins lucrativos, a qual contou com um investimento de 60 milhões de dólares em sua etapa de desenvolvimento.

A partir desta data, ambas as instâncias ofertam cursos massivos abertos on-line, juntamente com universidades de todo o mundo. Desde então, a plataforma Edx tem mantido um crescimento constante enquanto a seu número de usuários e cursos, atualmente a plataforma desde sua criação oferece mais de 1300 cursos, com mais de 10 milhões de usuários no mundo todo.

Além disso, a plataforma não oferece apenas cursos $M O O C$ em inglês, como também tem se dedicado a ampliar os serviços em espanhol para a América Latina e Caribe com mais de 113 cursos de língua espanhola oferecidos por instituições educacionais.

No caso do México, no ano 2015, a Secretaria de Educação (Ministério), através da Direção Geral de Televisão Educativa, iniciou os trabalhos de desenvolvimento de uma plataforma de código aberto, derivado do projeto $E d x$, juntamente com universidades e centros educacionais do país.

Deste modo, nasceu o projeto denominado MéxicoX, respaldado pela política pública Estrategia Digital Nacional (2012-2018), que foi instrumentada na reforma constitucional de telecomunicações, estabelecendo como meta aumentar os conteúdos de livre acesso para a população em geral.

Entre as primeiras instituições educacionais públicas e privadas de ensino superior que participaram do projeto, estiveram: o Colégio da Fronteira Norte, Colégio do México, Instituto Nacional de Saúde Pública, Instituto Politécnico Nacional, Tecnológico Nacional do México, Universidade Nacional Aberta e a Distância do México, Universidade Nacional Autônoma do México, Universidade Pedagógica Nacional, Universidade de Claustro de Sor 
Juana e a Direção Geral de Televisão Educacional, as quais iniciaram a produção e lançamentos dos primeiros 15 cursos.

Os cursos desenvolvidos na plataforma tiveram mais de 340 mil inscritos a nível mundial, atualmente a plataforma conta com mais de 2 milhões de usuários inscritos, em seus 160 cursos desenvolvidos por 61 instituições educacionais.

Como parte desta oferta educacional, no mês de maio de 2017 se realizou o curso MOOC Cátedra virtual Innovatic 2.0 como estratégia nacional para a geração de habilidades para jovens nos temas inovação e empreendedorismo.

O curso foi de caráter interinstitucional, onde participaram entidades governamentais por iniciativa da Confederação de Câmaras Industriais dos Estados Unidos Mexicanos (CONCAMIN) para fortalecer o público jovem de instituições de ensino superior às competências e habilidades em inovação e empreendedorismo, orientando a transformar uma ideia criativa em um negócio sustentável.

Os conteúdos do curso foram desenvolvidos por especialistas e pesquisadores em temas de educação e empreendedorismo das universidades nacionais de prestígio, principalmente a Universidade Nacional Autônoma do México (UNAM), o Instituto Politécnico Nacional (IPN), e Tecnológico Nacional do México (TNM) e a Universidade Veracruzana (UV); se estruturou em cinco módulos temáticos, com uma dedicação estimada de 40 horas em cinco semanas. Os módulos foram integrados em cinco tópicos:

1. Não precisa de um cérebro privilegiado para ser criativo. Sobre o pensamento criativo.

2. O futuro não se prevê, se constrói. Sobre o pensamento estratégico.

3. Se você sonha, é possível. Megatendências e solução de problemas.

4. Mãos à obra, exploremos. Desenvolvendo uma ideia de negócio.

5. O importante é innovar.

\section{Metodologia}

Realizou-se um estudo de campo (Kerlinger y Lee, 2002) de tipo avaliativo e descritivo que pretende contribuir com a medição e impacto da qualidade dos MOOC na formação das habilidades para a inovação e empreendedorismo nos jovens, como se destacou na seleção anterior, através de um instrumento de coleta de informações por questionário ou survey research (Creswell, 2008).

O propósito da pesquisa foi o diagnóstico dos usuários de $M O O C$, que permitiu conhecer suas características sociodemográficas e de interação com a plataforma, bem como o nível de satisfação pelos conteúdos do curso. 
A população do estudo compreendeu 28612 usuários e a aplicação da pesquisa se realizou de maneira aleatória a 3135 participantes mediante o serviço web.

\section{Resultados}

Foram registrados 28612 usuários do MOOC no período de 8 de setembro a 23 de outubro de 2017, dos quais 552 cancelaram sua inscrição (1.82 \%), 16455 (57.5\%) participantes foram homens e 12089 (42.2\%) mulheres. A idade média dos usuários foi 28.5 anos (Figura 1) e se concentrou principalmente na população de 21 a 25 anos (34\%).

Figura 1 - Idade dos usuários.

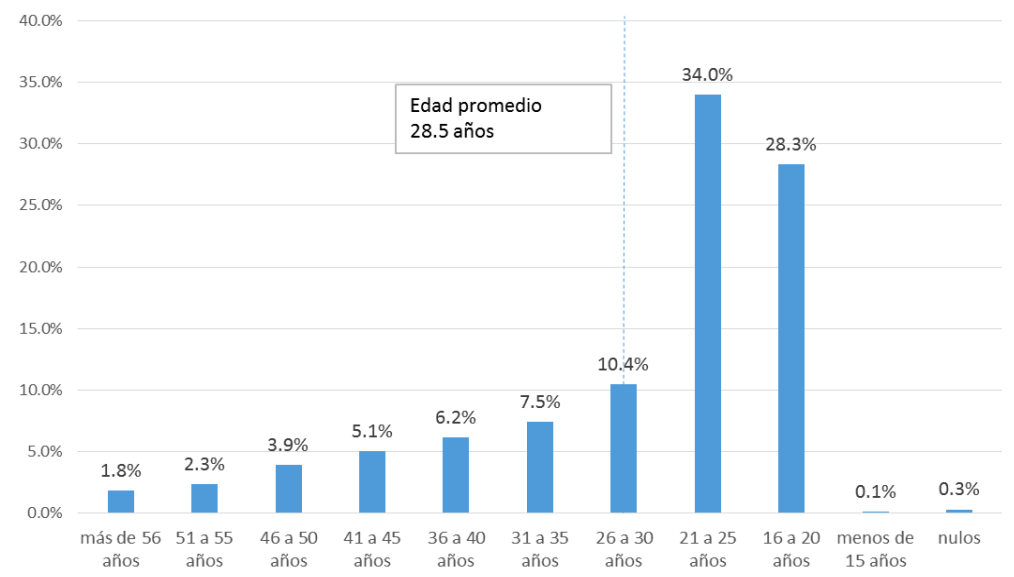

Fonte: Suporte técnico da Cátedra virtual InnovaTIC 2.0

O nível educacional dos participantes (Figura 2) correspondeu ao nível superior (70.5\%). Como se pode observar na Figura 3 mais da metade dos usuários foram estudantes (58.3\%) enquanto a participação dos docentes foi menor (9.8\%).

Figura 2 - Nivel educacional dos usuários. 


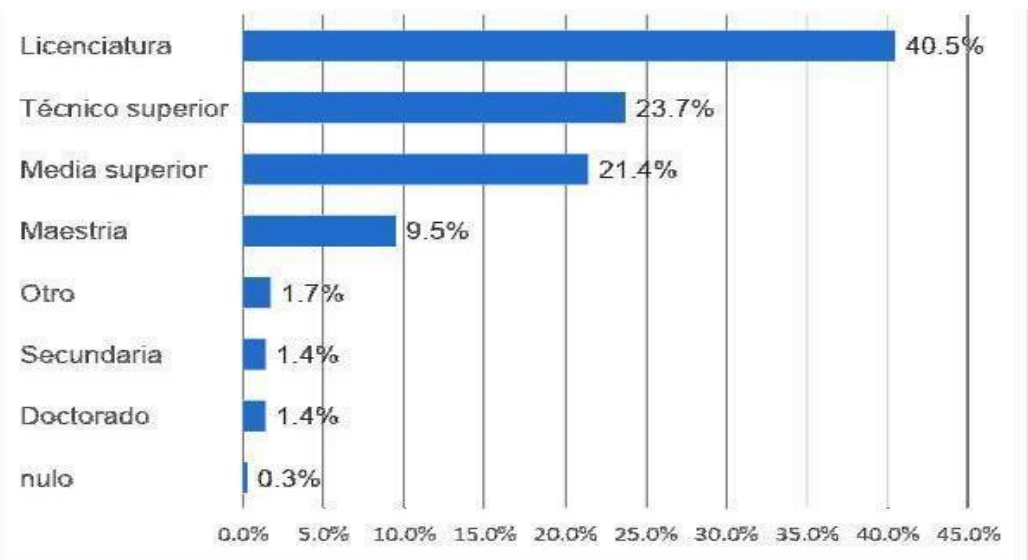

Fonte: Suporte técnico da Cátedra virtual InnovaTIC 2.0

Figura 3 - Usuários inscritos por ocupação.

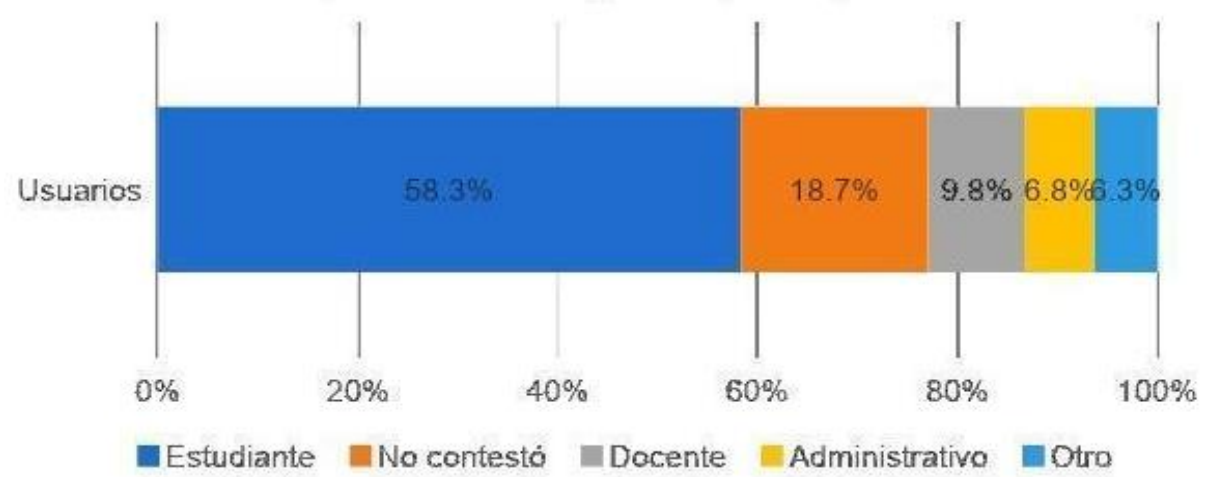

Fonte: Suporte técnico da Cátedra virtual InnovaTIC 2.0

Os usuários aumentaram sua participação (48.9\%) no curso à medida que este chegava a seu fim (Figura 4) e alcançou uma eficiência terminal de $22.92 \%$, ou seja, 5805 participantes concluíram o curso, por outro lado, 25436 não obtiveram a constância de acreditação da Cátedra Virtual. Numa escada de 1 a 10, considerando que a pontuação de 10 representa o máximo desempenho no $M O O C$, e a média mínima probatória se deu na pontuação 6 , mais de $21 \%$ dos usuários obtiveram uma pontuação igual a 8 ou superior (Figura 5).

Figura 5 - Atividade dos usuários na plataforma tecnológica. 


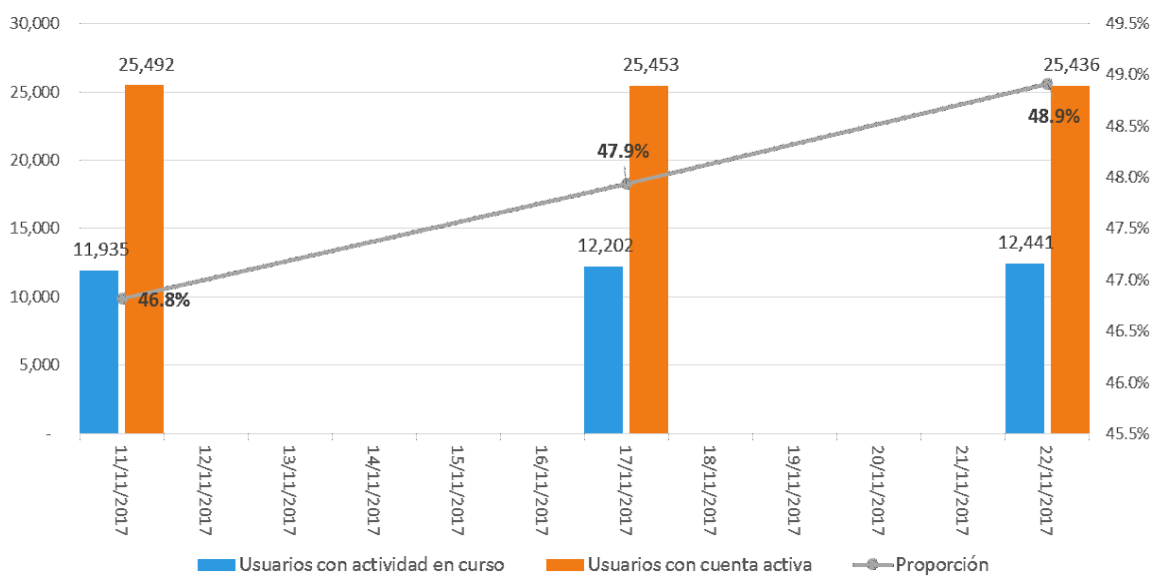

Fonte: Suporte técnico da Cátedra virtual InnovaTIC 2.0

Figura 5 - Qualificações médias no $M O O C$.

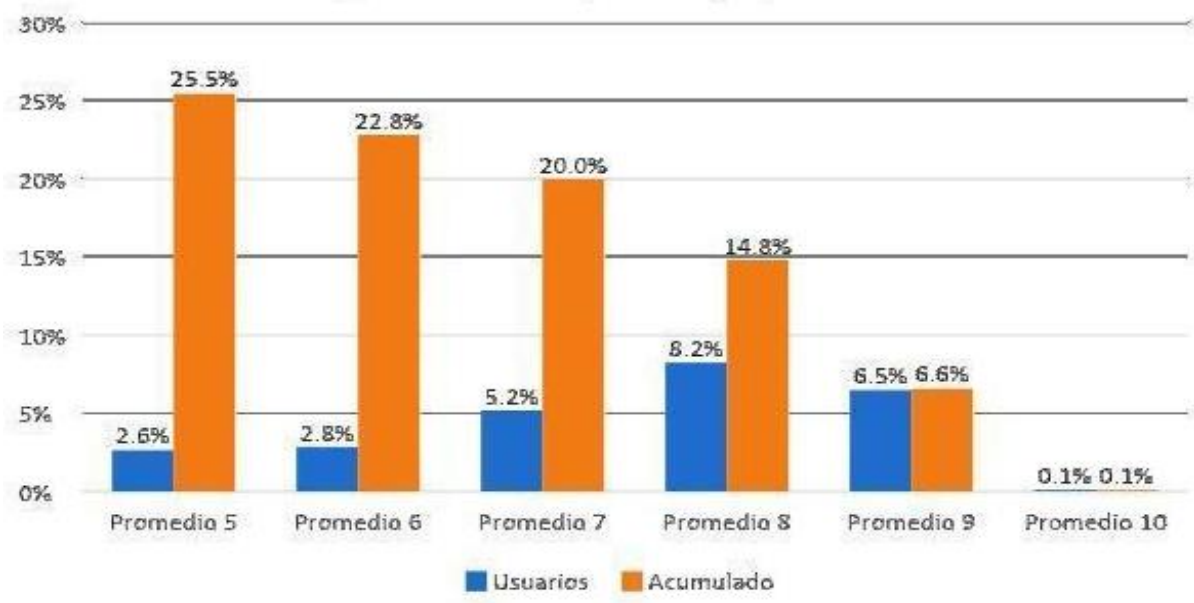

Fonte: Suporte técnico da Cátedra virtual InnovaTIC 2.0

\section{Pesquisa de satisfação}

Com a finalidade de evidenciar a participação dos participantes acerca dos conteúdos do curso, foi aplicada uma pesquisa de satisfação a todos os usuários da Cátedra virtual InnovaTIC 2.0 através de um formulário web.

A pesquisa foi compartilhada por e-mail e na página inicial do curso, onde se consideraram diferentes elementos, entre os quais se destacaram a difusão da cátedra virtual e a qualidade dos conteúdos (escrita, imagem e vídeo).

Sobre a difusão da cátedra, $60.3 \%$ se inteirou em sua escola, $15.5 \%$ através da divulgação de MéxicoX e $10 \%$ recebeu um convite por e-mail. Um total de $44 \%$ cursou a 
cátedra para fortalecer habilidades em inovação (Figura 6) e sobre a qualidade dos conteúdos, 92\% dos usuários os considerou interessantes.

Um total de $93.3 \%$ dos participantes concordou acerca da clareza de como se apresentaram os conteúdos na cátedra. O material audiovisual do MOOC foi considerado de utilidade por $94.2 \%$ dos participantes. Finalmente, 92\% dos usuários recomendariam a Cátedra para uma segunda emissão.

Figura 6 - Propósito de cursar cátedra.

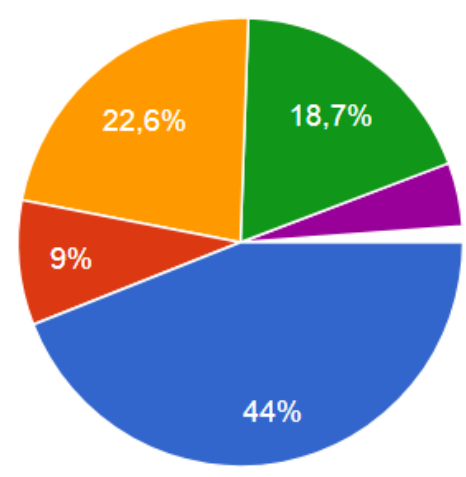

Para fortalecer mis habilidades en i...

Tenía ya una idea innovadora en m...

Me lo solicitaron en la escuela

Me interesaron los temas

Recomendación

Doy clases de secundaria, la materi...

Conocer el ámbito de la innovación.

por que si no me iban a reprobar

$1 / 6$

Fonte: Suporte técnico da Cátedra virtual InnovaTIC 2.0

\section{Considerações finais}

De acordo com a experiência da implementação do MOOC Cátedra Virtual Innovatic 2.0, se identificou uma grande demanda por campo de conhecimento de inovação e empreendimento, particularmente para o segmento de jovens no ensino superior, com respeito a isso, estamos de acordo com Jaramillo, Morales, Escobedo y Ramos (2013, p. 32), no que tange:

a idade do empreendedor é fator determinante para a capacidade das empresas de inovar, já que a medida que aumenta a idade do empreendedor, há uma maior propensão de que seja inovador, chegando a certo limite de idade, tal propensão diminui. ${ }^{4}$

Os participantes do curso mostraram interesse por culminar a experiência educacional, com base no índice da eficiência terminal, que para as modalidades de aprendizagem on-line e

${ }^{4}$ la edad del emprendedor es determinante de la capacidad de innovar de las empresas, ya que a medida que aumenta la edad del emprendedor, existe mayor propensión a que sea innovador llegado a cierto límite de edad en la cual dicha propensión disminuye. 
como indicador de qualidade educacional, se considera alto, com base em Billings (2014) ao referir que das limitações dos $M O O C$ é que não proporcionam a informação suficiente para identificar as variáveis que originam que aproximadamente $10 \%$, ou menos, dos participantes concluíam com êxito.

De acordo com o que foi dito, a planejamento pedagógico do programa, associado aos conteúdos educacionais e atividades de aprendizagem, representaram algumas dessas variáveis que poderiam explicar os resultados da referente eficiência terminal do $M O O C$, o que confirma a perspectiva de Capristán (2016) ao referir que "podemos ressaltar que a organização e o apoio que a equipe pedagógica recebe por parte da instituição para desenhar e implementar o MOOC é crucial" (p. 10).

Por outro lado, as alianças estratégicas entre os setores acadêmicos e os empresariais, construíram uma condição ou área de oportunidade para o desenvolvimento do projeto de inovação e empreendedorismo, bem como se constituíram como fator chave para a vinculação universidade-empresa.

A média de idade e perfil dos usuários nesta modalidade educacional a distância caracterizou uma classe de novos usuários de internet e de consumidores de produtos e serviços educacional baseadas na web 3.0, que pretendem o desenvolvimento de habilidades específicas para a formação acadêmica e profissional.

Por fim, a usabilidade e experiência dos usuários do $M O O C$ se relacionaram com um alto nível de satisfação, pelo qual as plataformas para a gestão e distribuição de conteúdos, baseadas em ambientes virtuais de aprendizagem, representam um potencial recurso pedagógico para sua incorporação na educação formal universitária, o qual conduz a uma valorização institucional das modalidades híbridas para a aprendizagem que combinem a educação presencial com a mediação das plataformas técno-educacionais, o que significa que “os modelos híbridos de aprendizagem (blended learning, b-learning) estão surgindo como uma inovação híbrida, como uma possibilidade de compromisso estudantil, inovação sustentável em comparação com a sala de aula tradicional" (MEJÍA; MICHALÓN; MICHALÓN; LÓPEZ; PALMERO; SÁNCHEZ, 2017, p. 351).

\section{REFERENCIAS}

AREA, M. Hacia la universidad digital: ¿dónde estamos y a dónde vamos? RIED. Revista Iberoamericana de Educación a Distancia, v. 21, n. 2, p. 25-30, 2018. Disponível em: http://revistas.uned.es/index.php/ried/article/view/21801. Acesso em: 27 fev. 2020. DOI: https://doi.org/10.5944/ried.21.2.21801 
BATES, A. W. (2015). La Enseñanza en la Era Digital. Una guía para la enseñanza y el aprendizaje. BC Campus. 2015. 521p.Disponível em: https://bit.ly/2Dexbff. Acesso em: 27 fev. 2020.

BILLINGS, D. M. Understanding massively open online courses. The Journal of Continuing Education in Nursing, v. 45, n. 2, p. 58-59, 2014. Disponível em: https://www.healio.com/nursing/journals/jcen/2014-2-45-2/\%7Bfb4b5ac7-42c2-4105-93671c08fe0095b5\%7D/understanding-massively-open-online-courses\#divReadThis. Acesso em: 27 fev. 2020. DOI: https://doi.org/10.3928/00220124-20140124-14.

CAPRISTÁN, B. Diseño instruccional en los MOOC: ¿qué aspectos tomar en cuenta? Revista Digital Universitaria. v. 17, n. 2, 1 fev. 2016. ISSN: 1607-6079. Disponível em: http://www.revista.unam.mx/vol.17/num2/art15/art15.pdf. Acesso em: 27 fev. 2020.

CASTAÑO, C.; GARAY, U.; THEMISTOKLEOUS, S. De la revolución del software a la del hardware en educación superior. Revista Iberoamericana de Educación a Distancia, v.21, n. 1, p. 135-153, 2018. Disponível em: http://revistas.uned.es/index.php/ried/article/view/18823. Acceso en: 27 fev. 2020. DOI: http://dx.doi.org/10.5944/ried.21.1.18823

CLARK, D. MOOCs: taxonomy of 8 types of MOOC. Donald Clark Plan B. 2013. Disponível em: http://donaldclarkplanb.blogspot.com/2013/04/moocs-taxonomy-of-8-typesof-mooc.html. Acceso en: 27 fev. 2020.

CRESWELL, J. W. (2008). Educational research: Planning, conducting, and evaluating quantitative and qualitative research. Nueva Jersey, EUA: Pearson/Merrill Prentice Hall. 2008.

EDEL, RUBÉN.; NAVARRO, Y. Entornos virtuales de aprendizaje 2002- 2011. México: ANUIES. 2015.

GARDUÑO, R. Las Tecnologías y la Educación Superior a Distancia en México. Revista Digital Universitaria, v. 9, n. 9, 10 set. 2008. México: UNAM. Disponível em: http://www.revista.unam.mx/vol.9/num9/art64/art64.pdf. Acesso em: 27 fev 2020.

JARAMILLO, J. L.; MORALES, J.; ESCOBEDO, J.S.; RAMOS, J.G. (2013). Factores que influyen para el emprendimiento de microempresas agropecuarias en el Valle de Puebla, México. Revista mexicana de ciencias agrícolas, v. 4, n. esp. 5, p. 925-937, maio/jun. 2013. Disponível em: http://www.scielo.org.mx/scielo.php?script=sci_arttext\&pid=S200709342013000900006\&lng=es\&tlng=es. Acesso em: 27 fev 2020.

KERLINGER, F. N.; LEE, H. B. Investigación del comportamiento. Métodos de investigación en ciencias sociales. México: McGraw-Hill. 2002.

MEJÍA, C.; MICHALÓN, D.; MICHALÓN, R.; LÓPEZ, R.; PALMERO, D.; SÁNCHEZ, S. Espacios de aprendizaje híbridos. Hacia una educación del futuro en la Universidad de Guayaquil. Revista electrónica Medisur, v. 15, n. 3, p. 350-355, jun. 2017. Disponível em: http://www.medisur.sld.cu/index.php/medisur/article/view/3605. Acesso en: 27 fev 2020. 
Stiglitz J.E. y Greenwald B. E. La creación de una sociedad del aprendizaje. México: Paidós. 2014.

TORRES, D.; GAGO, D. Los MOOCs y su papel en la creación de comunidades de aprendizaje y participación. Revista Iberoamericana de Educación Superior a Distancia, v. 17, n. 1, p. 13-34, 2014. Recuperado de:

http://revistas.uned.es/index.php/ried/article/view/11570. Acesso en: 27 fev 2020. DOI:

https://doi.org/10.5944/ried.17.1.11570

VALVERDE, J. (2014). MOOCS: Una visión crítica desde las ciencias de la educación.

Revista de Curriculum y Formación de Profesorado. v. 18, n. 1, p. 93-111, jan./abr. 2014.

Disponível em:_https://recyt.fecyt.es/index.php/profesorado/article/view/41070. Acesso em:

27 fev 2020.

ZUBIETA, J.; RAMA, C. La Educación a Distancia en México: Una nueva realidad universitaria. México: UNAM, v. 7, n. 14, p. 155-156, 7 ago. 2015. Disponível em: http://revistas.unam.mx/index.php/rmbd/article/view/65264. Acesso en: 27 fev 2020. DOI: http://dx.doi.org/10.22201/cuaed.20074751e.2015.14.65264

\section{Como referenciar este artigo}

NAVARRO, Rubén Edel; MÉNDEZ, Germán Ruiz; AGUILAR, Genaro Aguirre. Mediação tecnológica e caracterização de moocs: uma abordagem da Cátedra Virtual Innovatic 2.0. Revista Ibero-Americana de Estudos em Educação, Araraquara, v. 15, n. 2, p. 347-360, abr./jun. 2020. e-ISSN: 1982-5587. DOI: https://doi.org/10.21723/riaee.v15i2.12287

Submetido em: 20/02/2019

Revisões requeridas: 30/09/2019

Aprovado em: 20/12/2019

Publicado em: 20/02/2020 